\title{
Neovascularization in Advanced Atherosclerosis - A Histomorphometric Study
}

\author{
VS Veena Paul ${ }^{1}$, PP Christina Mary ${ }^{2}$, Kuruvilla Sarah ${ }^{3}$ \\ ${ }^{1}$ Dr Veena Paul VS, MBBS, MD (Pathology), Assistant Professor of Pathology, A.C.S Medical College, \\ Velappanchavadi, Chennai, ${ }^{2}$ Dr. Christina Mary Priya Paul, M.B.B.S., M.D (Community Medicine), Associate Professor \\ of Community Medicine, A.C.S Medical College, Chennai, ${ }^{3}$ Dr. Sarah Kuruvilla, M.B.B.S., M.D, (Pathology), DNB, \\ MNAMS, FIC (Path), Senior Consultant \& Head of the Department of Pathology, The Madras Medical Mission Hospital, \\ Chennai, India.
}

Address for Correspondence: Dr. Veena Paul V. S., M.B.B.S., M.D (Pathology), Assistant Professor of Pathology, A.C.S. Medical College, Velappanchavadi, Chennai, e-mail: veronica212121@yahoo.com

\begin{abstract}
Introduction: Neovascularization is an important component of advanced atherosclerosis which leads to plaque vulnerability and rupture. This is an endarterectomy study where neovascularization is quantified in 50 advanced atherosclerotic plaques by using IHC stain and measuring the positivity by histomorphometry, simultaneously assessing lipid pool area and fibrous cap thickness (average and minimum), also by histomorphometry. The values were compared across different AHA types and also probable vulnerable plaques were identified. Materials \& Methods: Three heart specimens obtained at autopsy from subjects who died of RTA showed evidence of coronary artery disease on postmortem coronary angiogram. Coronary endarterectomy was done from these specimens and was made into tissue blocks for histological study. On H\& E sections the plaques were classified by AHA typing and 50 advanced atherosclerotic plaques were chosen for this study. To assess neovascularization, the IHC stain CD34 was used and the percentage area of positivity was later measured using histomorphometry. The lipid pool area in the plaques and the fibrous cap thickness were also measured using histomorphometry. Results: In our study, 47 out of 50 advanced lesions showed neovascularization although the difference in their amounts between AHA Type IV and V was not statistically significant. On measuring lipid pool area and fibrous cap thickness, we found 4 of the 50 plaques to be probable vulnerable plaques as they had a combination of a large lipid pool (>40\% of the plaque area) and a thin fibrous cap $(<65 \mu)$ in addition to revascularization. Conclusion: This study showed that neovascularization was seen in most of the advanced atherosclerotic plaques and together with some other features like large lipid pool and thin fibrous cap, it can potentially make a plaque vulnerable to rupture. The understanding of role of neovascularization in plaque rupture has led to the trial of antiangiogenic therapy to prevent plaque progression.
\end{abstract}

Keywords: Neovascularization in Atherosclerosis, Vulnerable Plaque, Histomorphometry in Atherosclerotic plaques.

\section{Introduction}

Atherosclerosis is a disease of large and medium-sized muscular arteries and is characterized by endothelial dysfunction, vascular inflammation, and the buildup of lipids, cholesterol, calcium, and cellular debris within the intima of the vessel wall. These build-up results in plaque formation, vascular remodeling, acute and chronic luminal obstruction, abnormalities of blood flow and diminished oxygen supply to target organs [1]. More than $90 \%$ of all coronary artery diseases, the

Manuscript received: $4^{\text {th }}$ Jan 2016

Reviewed: $22^{\text {nd }}$ Jan 2016

Author Corrected: $02^{\text {nd }} \mathrm{Feb} 2016$

Accepted for Publication: $12^{\text {th }}$ Feb 2016
Leading cause of death all over the world, are caused by atherosclerosis. In atherosclerosis, the formation of new vessels around the arterial wall can be seen even before the development of endothelial dysfunction and plaque formation. The newly formed vessels are leaky and fragile and for this reason may lead to the extravasation of RBCs and even true intraplaque hemorrhage. Given the rich cholesterol content of RBCs, this may contribute to the lipid loading of the atherosclerotic plaque, its progression in volume, and vulnerability. Plaque neovascularization seems to characterize not only the vulnerable plaque but also the vulnerable 
patient. Angiogenesis is an associate of atherosclerosis through its various stages [2].Most of the studies on atherosclerosis are either autopsy studies or studies on experimental animals as it is difficult to obtain atherosclerotic tissue from live patients. This is a study on endarterectomy specimens obtained at autopsy from subjects who died of road traffic accidents who had evidence of coronary artery disease on post-mortem coronary angiogram. This study is aimed at quantifying neovascularization in advanced atherosclerotic plaques by staining with the immunostain CD34 and later measuring the percentage area of positivity using histomorphometry. Together with neovascularization, other parameters like lipid pool area and fibrous cap thickness which are also factors that make a plaque vulnerable to rupture, are also measured in advanced atherosclerotic lesions in this study and are compared between different AHA types.

\section{Materials \& Methods}

Three heart specimens were collected from autopsies done for cases of accidental deaths. The heart specimens were perfusion-fixed and post mortem coronary angiogram [3] was performed in all three specimens. Post mortem clots were flushed out by infusing saline at $38^{\circ} \mathrm{C}$. The arteries were then perfused with an Iso-opaque dye. The findings were recorded on $\mathrm{X}$-ray angiograms in both anterior and lateral views. The four main coronary arteries were dissected intact from the specimen. The coronary arteries were then divided into $5 \mathrm{~mm}$ transverse slices throughout the entire length. Each slice was further fixed in formalin and was embedded in paraffin wax blocks.

Then each section was examined using H \& E stain for the presence of plaque and the number of plaques in each vessel was noted. Then the plaques were classified according to the American Heart Association (AHA) classification and early lesions (AHA Type I, II and III) [4] were separated from the advanced lesions (AHA Type IV and above) [5]. 50 advanced atherosclerotic plaques based on the morphology on $\mathrm{H} \& \mathrm{E}$ sections (Fig. 1) were chosen for this study and were stained with the immunostain CD34 to highlight the neovascularization in the plaque. Each batch of the immunohistochemical staining of the test samples were done with appropriate positive and negative controls to ensure the test results were valid and accurate. Tissue sections from a case of Dermatofibrosarcoma protruberans was used as the positive control (Fig. 2) for the CD34 stain. Then, the percentage-area of CD34 positivity in the plaques was measured using histomorphometry. The percentage-area of lipid pool and the thickness of fibrous cap (average and minimum thickness) were also measured using histomorphometry on H\&E sections.

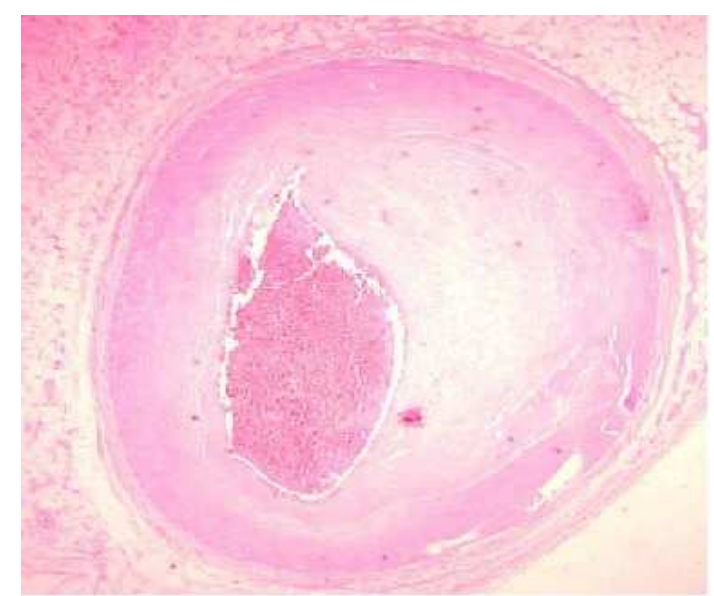

Fig 1: Scanner view of vessel with AHA Type IV plaque with outward remodeling H \& E stain, x 200

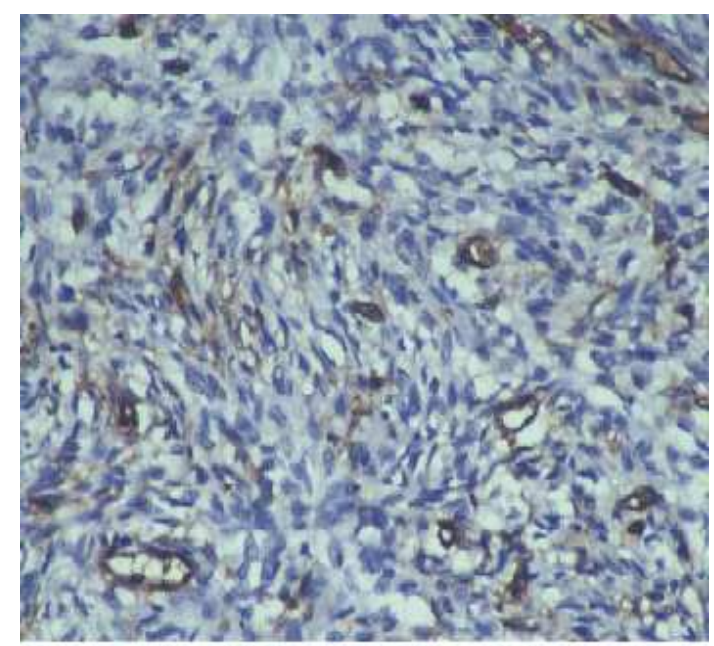

Fig. 2: High power view of CD34 immunostain in a sindle-cell neoplasm (Positive control) x 400

\section{Results}

On post-mortem, coronary angiogram, all 3 specimens showed evidence of triple vessel disease. On histomorphological classification as per the American Heart Association typing, the plaques ranged from AHA types II to V. Among these, 50 plaques of AHA Type IV and V (advanced lesions) were chosen and were stained with CD34, the immunohistochemical stain for endothelial cells to study the angiogenesis within the plaque. CD34 staining for neovascularization identified plaque angiogenesis in 47 out of 50 plaques (Fig. $3 \& 4$ ). Quantification of neovascularization by 
measuring percentage area of CD34 staining by histomorphometry did not have any statistically

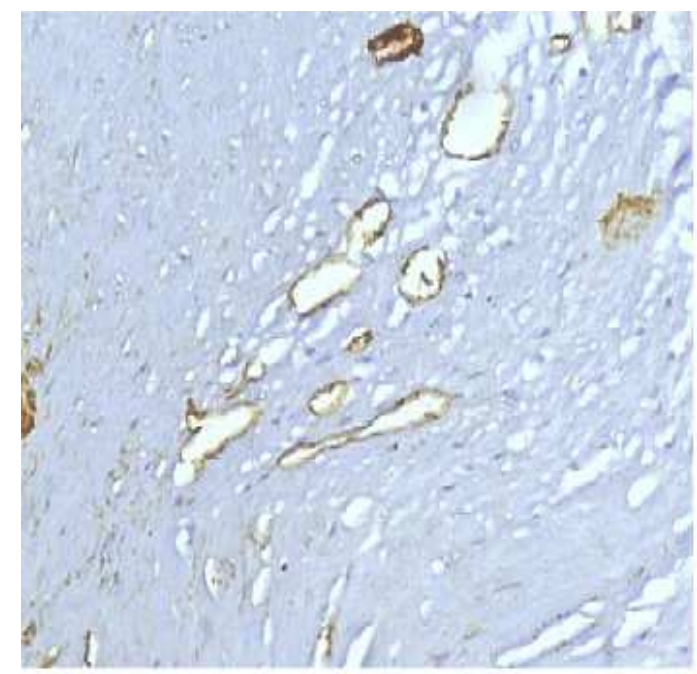

Fig 3: Low power view of CD34 immunostain in the plaque showing neovascularization $\mathrm{x} 100$ significant difference between AHA Type IV and AHA Type V lesions (Table 1).

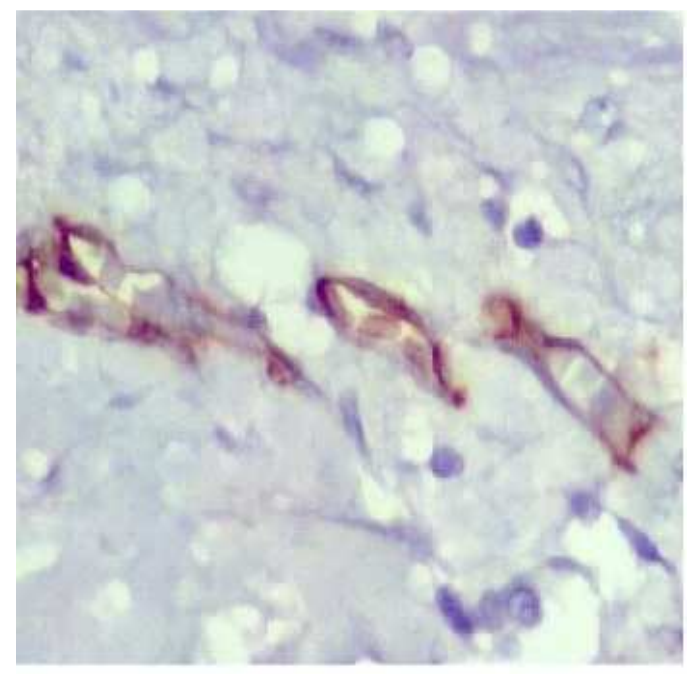

Fig. 4: High power view of CD 34 stain in the plaque showing cytoplasmic positivity in the endothelial cells x 400

Plaques with a large lipid pool (>40\%), [6] together with a thin fibrous cap and some other predisposing factors like neovascularization and inflammation were separated from all three specimens as they form potential vulnerable plaques. It was observed that 7 out of 50 plaques had large lipid pools of which 4 were AHA type V and were AHA type IV plaques. Similarly, the thickness of fibrous cap was measured using histomorphometry and the average and minimum thickness was noted for all plaques. Plaques with a thin fibrous cap $(<65 \mu)$ [7] were separated out from both AHA type IV and type $\mathrm{V}$ lesions. It was noted that the difference in average thickness and minimum thickness among AHA type IV and type $\mathrm{V}$ lesions was not statistically significant (Table 1). It was observed that 6 out of 50 plaques had a thin fibrous cap of which 4 were Type IV and 2 were type V AHA lesions.

Table 1: The results of all comparative statistical tests using Epinfo6.04d for Dos (Tests done for alpha 0.5, power$80 \%, 2$-sided test)

\begin{tabular}{|l|l|l|}
\hline Comparisons & 't' statistic value & p-value \\
\hline AHA Type Vs CD34 \% Area & 1.23 & 0.22 \\
\hline AHA Type Vs Lipid Pool \% Area & 1.74 & 0.08 \\
\hline AHA Type Vs Fibrous Cap Thickness (Average) & 0.38 & 0.7 \\
\hline AHA Type Vs Fibrous Cap Thickness (Minimum) & 1 & 0.3 \\
\hline
\end{tabular}

Plaques with a combination of both large lipid pool and a thin fibrous cap were then separated out as they might constitute a vulnerable plaque together with other factors like inflammation, lumen stenosis etc. In our study it was observed that there were 4 out of 50 plaques that had a combination of large lipid pool and thin fibrous cap of which 2 were of AHA type IV and 2 were of AHA type V. These plaques were considered as probable vulnerable plaques.

\section{Discussion}

Ectopic neovascularizationin the intima and media is a hallmark of advanced atherosclerotic lesions [8]. Given the early onset of vasa vasorum neovascularization in atherogenesis, factors other than hypoxia, classically thought to be the main stimulus for angiogenesis, have to be considered. Increased oxidative stress in the arterial wall may stimulate vasa vasorum neovascularization [9]. Inflammation may be another important factor, and there may even be a mutual interaction between vascular inflammation and neovascularization. As the atherosclerotic lesion increases in volume, hypoxia may become a more 
prominent stimulus for neovessel formation. At that point, further growth of the atherosclerotic plaques may actually depend on angiogenesis, reminiscent of a cancerous lesion. Moreover, vasa vasorum neovascularization evolves mainly in the area of intimal thickening, indicating cross-talk between the intima and the adventitia. Indeed, this neovascularization process creates a potential entry port for inflammatory and proliferative factors, red blood cells (RBCs), and inflammatory cells from the circulation to the adventitia.

In this process, the media and subintimal space become sandwiched between two highly vascular layers and directly exposed to an extensive endothelial surface area on either side. As the atherosclerotic plaque develops, neovessels sprout from the adventitial vasa vasorum through the media into the intima lesion. Only a small fraction of the intima vessels can be traced back to the main lumen. The plaque areas that are particularly rich in neovessels include the shoulder region and the base.

The majority of microvessels arise from the adventitial vasa vasorum and rarely from the luminal surface of the parent artery [10]. Moreno PR et al demonstrated that microvessel density is greatest in lesions with marked macrophage infiltration of the fibrous cap. They correlated presence of angiogenesis at the base of the plaque independently with plaque rupture underscoring the potential for a direct contributory role of neovascularization in this process [6]. These pathological microvessels are prone to rupture and intraplaque hemorrhage has shown to accelerate plaque formation [11]. As an additional factor, release of free hemoglobin can stimulate oxidative stress and inflammation in the

atherosclerotic plaque. The newly formed vessels may contribute to these unfavorable plaque dynamics further by serving as conduits for inflammatory cells and soluble factors [12]. Moreover, proteolytic enzyme activity during the neovascularization process may weaken plaque structures, contributing to plaque vulnerability, especially in the shoulder regions. Thus, the microvasculature of the plaque may contribute to the progression and complication of atherosclerotic disease and may therefore be of therapeutic interest.

A vulnerable plaque is one which is susceptible to producing complications in a vulnerable patient [13]. A vulnerable plaque is defined based on some criteria such as active inflammation with macrophages or $\mathrm{T}$ cells, thin fibrous cap $(<65 \mu)$ with a large lipid core ( $>40 \%$ of the plaque volume), stenosis $>90 \%$ etc [6]. A thin cap fibroatheroma is more prone to rupture as against a stable fibroatheroma as confirmed by studies by RenuVirmani et al. [7]. Plaque rupture is the most common type of plaque complication accounting for about $70 \%$ of fatal acute myocardial infarctions and/or sudden coronary deaths.

In our study, it was observed that $94 \%$ of the advanced atherosclerotic plaques showed intimal neovascularization, though of varying degrees. There was no statistically significant difference between the amount of neovascularization in plaques belonging to AHA Types IV and V. Lipid pool content and fibrous cap thickness (average and minimum) compared between AHA type IV and Type V lesions did not show a statistically significant difference. We also identified 4 probable vulnerable plaques among the 50 advanced lesions examined as they had both large lipid pool area and a thin fibrous cap in addition to neovascularization.

\section{Conclusion}

Thus, intimal neovascularization was observed in most of the advanced lesions of atherosclerosis. There was no difference in the amount of neovascularization between AHA type IV and type V. This study has its limitations because of the small sample size and methods such as morphometry for measuring percentage area as it only measures a portion of the plaque and not the entire plaque. However, it provides some useful data in the form of inter-plaque comparison and inter-AHA type comparison of features such as neovascularization, lipid pool area and fibrous cap thickness which are important factors that influence the vulnerability of a plaque to rupture. The understanding of neovascularization and its role in plaque hemorrhage and rupture has led to trial of antiangiogenic therapy [10] to prevent plaque progression.

Funding: Nil, Conflict of interest: None. Permission of IRB: Yes

\section{References}

1. Libby P. Vascular disease, Atherosclerosis; in: Fauci, Braunwald, Isselbacher, Wilson, Martin, Kasper, Hauser, Longo. eds. Harrison's Principles of Internal Medicine, New York McGraw Hill companies; 14th edition 1998: 1345-52. 
2. Herrmann J, Lerman A. Atherosclerosis in the back yard. J Am Coll Cardiol. 2007 May 29; 49(21):2102-4. Epub 2007 May 17.

3. Coghill SB, Nicoll SM, McKimmie A, Houston I, Matthew BM. Revitalising postmortem coronary angiography. J Clin Pathol. 1983 Dec;36(12):1406-9. doi:10.1136/jcp.36.12.1406.

4. Stary HC, Chandler AB, Glagov S, Guyton JR, Insull, Jr, W, Rosenfeld ME, Schaffer SA, Schwartz CJ, Wagner WD, Wissler . A definition of initial, fatty streak, and intermediate lesions of atherosclerosis. A report from the Committee on Vascular Lesions of the Council on Arteriosclerosis, American Heart Association. Circulation 1994 May;89(5):2462-78. doi: 10.1161/01.CIR.89.5.2462.

5. Stary HC, Chandler AB, Dinsmore RE, Fuster V, Glagov S, Insull, Jr, W, Rosenfeld ME, Schwartz CJ, Wagner WD, Wissler RW. Definition of Advanced Types of Atherosclerotic Lesions and a Histological Classification of Atherosclerosis. Circulation 1995; 92:1355-1374. doi: 10.1161/01.CIR.92.5.1355.

6. Naghavi M, Libby P, Falk E, Casscells SW, Litovsky $\mathrm{S}$, Rumberger $\mathrm{J}$, et al. From Vulnerable Plaque to Vulnerable Patient: A Call for New Definitions and Risk Assessment Strategies: Part I. Circulation 2003;108(15):1664-1672.

doi: 10.1161/01.CIR.0000087481.55887.C9.

7. Virmani R, Kolodgie FD, Burke AP, Farb A, Schwartz SM. Lessons From Sudden Coronary Death: A Comprehensive Morphological Classification Scheme for Atherosclerotic Lesions. Arterioscler Thromb Vasc Biol. 2000 May;20(5):1262-1275. doi: 10.1161/01.ATV.20.5.1262.
8. Fleiner M, Kummer M, Mirlacher M, Sauter G, Cathomas G, Krapf R, Biedermann BC. Arterial neovascularization and inflammation in vulnerable patients: early and late signs of symptomatic atherosclerosis. Circulation. 2004 Nov 2;110(18):284350. Epub 2004 Oct 25.

9. Stary HC, Blankenhorn DH, Chandler AB, Glagov S, Insull, Jr, W, Richardson M, Rosenfeld ME, Schaffer SA, Schwartz CJ, Wagner, Jr, WD, Richardson M. A definition of the intima of human arteries and of its atherosclerosis- prone regions. A report from the Committee on Vascular Lesions of the Council on Arteriosclerosis, American Heart Association. Circulation 1992 Jan;85(1):391-405. doi:10.1161/01.CIR.85.1.391.

10. Doyle B, Caplice N. Plaque neovascularization and antiangiogenic therapy for atherosclerosis. J Am Coll Cardiol. 2007 May 29;49(21):2073-80. Epub 2007 May 17.

11. Kolodgie FD, Gold HK, Burke AP, Fowler DR, Kruth HS, Weber DK, Farb A, Guerrero LJ, Hayase M, Kutys R, Narula J, Finn AV, Virmani R. Intraplaque hemorrhage and progression of coronary atheroma. N Engl J Med. 2003 Dec 11;349(24):231625. doi: 10.1056/NEJMoa035655.

12. Packard RR, Libby P. Inflammation in atherosclerosis: from vascular biology to biomarker discovery and risk prediction. Clin Chem. 2008 Jan;54(1):24-38.

13. Moreno PR, Purushothaman KR, Fuster V, Echeverri D, Truszczynska H, Sharma SK, Badimon JJ, O'Connor WN. Plaque Neovascularization Is Increased in Ruptured Atherosclerotic Lesions of Human Aorta Implications for Plaque Vulnerability. Circulation 2004 Sep;110(14):2032-2038. doi: 10.1161/01.CIR.0000143233.87854.23.

\section{How to cite this article?}

VS Veena Paul, PP Christina Mary, Kuruvilla Sarah. Neovascularization in Advanced Atherosclerosis - A Histomorphometric Study. Int J Med Res Rev 2016;4(2):164-168. doi: 10.17511/ijmrr.2016.i02.007. 